\title{
$\operatorname{arCOS} D E S I G N$
}

\section{Relevância da comunicação visual na auto- administração de medicamentos por pacientes crônicos: Uma abordagem ergonômica}

Vera Lopes de Abreu Lima (PUC-Rio, Brasil)

moraergo@rdc.puc.br

Rua Marquês de São Vicente, 225, Gávea

Rio de Janeiro, RJ, Brasil, CEP: 22451-900

Anamaria de Moraes (in memorian) 


\title{
Relevância da comunicação visual na auto-administração de me- dicamentos por pacientes crônicos: Uma abordagem ergonômica
}

Resumo: O trabalho aqui apresentado tem como base parte da pesquisa de doutorado Comunicação na Utilização de Medicamentos por Pacientes Crônicos: uma Abordagem Ergonômica, desenvolvida entre os anos de 2008 e 2012. No presente artigo é investigada a relevância da comunicação visual na auto-administração de medicamentos por pacientes crônicos. Inicialmente são apresentados dados a respeito do aumento de enfermidades crônicas e da importância de se adequar o cuidado a estes pacientes. Em seguida são apresentados e discutidos os resultados obtidos por meio de um questionário respondidos por 35 pacientes com cardiopatia crônica residentes na cidade do Rio de Janeiro. Conclui-se que, para identificar os diferentes medicamentos prescritos, os pacientes fazem uso de diversos elementos gráficos presentes nas embalagem dos medicamentos (caixas e cartelas), receita médica e comprimido. Entretanto destaca-se a relevância das características dos comprimidos - forma, cor, tamanho, e demais atributos visuais identificados pelo paciente - na comunicação visual presente na auto-administração dos medicamentos.

Palavras-chave: ergonomia, auto-administração de medicamentos, comunicação visual na utilização de medicamentos

\section{Significance of visual communication at medication self-mana- gement by chronic diseases patients: An ergonomic approach}

\begin{abstract}
This study is based on part of the doctoral research Communication at Drug Utilization by Chronic Patients: An Ergonomic Approach, developed between the years 2008 and 2012 under the supervision of Professor Anamaria de Moraes. In this article we investigate the importance of visual communication at drug self-administration by chronic patients. First, it is presented data on chronic diseases incidence in Brazil and the importance of adapting health care to those patients. Then, it is presented and discussed the results of a questionnaire answered by 35 cardiovascular patients living in Rio de Janeiro city. Results indicate that, in order to distinguish the drugs taken, patients make use of many graphic elements present in medicines packaging (boxes and blisters), medical prescription and pills. It stands out the relevance of pill's attributes - shape, colour, size, and other visual characteristics at medication identification by patients.
\end{abstract}

Key words: ergonomics, medication self-management, visual communication at drug utilization 


\section{Introdução}

\subsection{Aumento de doenças crônicas}

A população brasileira vem sofrendo um rápido processo de envelhecimento. Segundo dados do Instituto Brasileiro de Geografia e estatística - IBGE, a porcentagem dos brasileiros com 14 anos ou menos, que em 1940 representavam $42,6 \%$ da população, será de apenas $13,1 \%$ no ano de 2050. Já a população idosa, que no ano de 1940 representava 4,1\% do total de brasileiros, passou para 10\% da população em 2010 e deve chegar a 29,8\% em 2050.

A esta transição demográfica está associado um processo de transição epidemiológica. Acompanhando a diminuição da população brasileira mais jovem, há a redução dos óbitos causada por doenças infectocontagiosas. Seguindo o aumento do número de idosos, ocorre uma maior incidência de morbidades crônicas. Somente as doenças cardiovasculares, que em 1950 representavam $12 \%$ das mortes no país, foram, em 2008, responsáveis por $40 \%$ dos óbitos. (Brasil, 2009).

Apesar da mudança ocorrida no perfil epidemiológico, o modelo de atendimento em saúde ainda é voltado para o cuidado agudo, apresentando barreiras à adesão no tratamento de doenças crônicas. (oMs, 2011, p.11).

Isto ocorre pelo fato de os dois tipos de cuidado em saúde - agudo e crônico apresentarem diferenças em fatores essenciais, conforme apresentado na tabela abaixo:

\begin{tabular}{|c|c|c|}
\hline & Cuidado agudo & Cuidado crônico \\
\hline $\begin{array}{l}\text { Objetivo principal do } \\
\text { tratamento }\end{array}$ & Cura & $\begin{array}{c}\text { Controle da doença; } \\
\text { Prolongamento da vida; } \\
\text { Aumento da qualidade de vida. }\end{array}$ \\
\hline Duração & Limitada & $\begin{array}{l}\text { Período longo, indefinido, } \\
\text { ou por toda a vida. }\end{array}$ \\
\hline Conhecimento & $\begin{array}{l}\text { Centrado nos } \\
\text { profissionais } \\
\text { de saúde. }\end{array}$ & $\begin{array}{l}\text { Profissionais de saúde. } \\
\text { Pacientes e familiares compartilham } \\
\text { conhecimento complementar. }\end{array}$ \\
\hline $\begin{array}{l}\text { Tratamento } \\
\text { da doença }\end{array}$ & $\begin{array}{l}\text { Focada no tratamento } \\
\text { agudo } \\
\text { e unicamente médico }\end{array}$ & $\begin{array}{l}\text { Estratégia multimedicamentosa e de } \\
\text { autocuidado/auto-gestão, com sistema } \\
\text { de saúde apropriado, apoio da família } \\
\text { e da comunidade. É comum a presença } \\
\text { de comorbidade. }\end{array}$ \\
\hline Provedor de saúde & $\begin{array}{l}\text { Normalmente clínicos } \\
\text { e instituições clínicas }\end{array}$ & $\begin{array}{l}\text { Largo espectro de serviços de saúde, } \\
\text { serviços à comunidade e saúde da } \\
\text { família. }\end{array}$ \\
\hline $\begin{array}{l}\text { Qualidade da } \\
\text { assistência }\end{array}$ & $\begin{array}{c}\text { Normalmente } \\
\text { abordagens } \\
\text { autossuficientes restritas } \\
\text { a cada instituição. }\end{array}$ & $\begin{array}{l}\text { Relevância de uma abordagem de } \\
\text { qualidade sistêmica. }\end{array}$ \\
\hline
\end{tabular}

Tabela 1. Comparação dos modelos de cuidado agudo e crônico. Fonte: Organização Mundial de Saúde. (Lima, 2012 apud oms, 2001). 
Destaca-se no tratamento crônico a relevância do paciente, família e comunidade como elementos determinantes no tratamento, o que não acontece no cuidado agudo, assim como a auto-gestão do tratamento. (oms, 2001).

A auto-administração - característica essencial do tratamento das enfermidades crônicas - é definida por Lorig (1993) como "o aprendizado e a prática das capacidades necessárias para conduzir uma vida ativa e emocionalmente satisfatória diante de uma doença crônica" (Lorig, 1993, p.1 tradução nossa). Bodenheimer e Grumbach (2007) acrescentam que a auto-administração — ou seja, a tomada diária de decisões a respeito de seus tratamentos - ocorre ainda que o paciente não esteja adequadamente preparado para esta tarefa. Os autores apresentam ainda o conceito de auto-eficácia, que é a "confiança para saber realizar o comportamento adequado necessário para alcançar o objetivo desejado" (BODENHIMER; GRUMBACH, 2007, p79, tradução nossa).

Malta e Merhy corroboram os autores acima e ressaltam a relevância da autonomia do paciente no tratamento de enfermidades crônicas:

"outro elemento-chave consiste em fortalecer a capacidade de o usuário cuidar de si, o que vai muito além de um simples autocuidado protocolado dos portadores de DCNT [doenças crônicas não transmissíveis], estimulando seu empoderamento, suas escolhas, suas opções." (MALta e MERYw, 2010, p. 250)

\subsection{Assistência Farmacêutica e utilização de medicamentos}

A assistência farmacêutica é uma política de saúde pública que tem o medicamento como insumo essencial para a proteção, promoção e recuperação da saúde individual ou coletiva. (Brasil, 1998). Ela ocorre ciclicamente, tendo como etapas: seleção, suprimento, armazenamento, distribuição e utilização de medicamentos. (Oliveira; Bermudes; Osório de Castro, 2007).

É na etapa de utilização que ocorre interação entre a assistência farmacêutica e o paciente. Esta etapa é composta por um outro ciclo, composto de: prescrição, dispensação e uso, descritos a seguir:

- prescrição - Etapa em que deve ocorrer a interação com o profissional de saúde prescritor - médico ou odontólogo. É a partir do entendimento entre profissional de saúde e paciente que é elaborada a prescrição médica

- dispensação - No atendimento ambulatorial, é a etapa em que deve ocorrer a entrega os medicamentos ao paciente, assim como informação e orientação sobre seu uso. (Peppe; Osório de Castro, 2011). 
- uso - Etapa onde são administradas as doses e também quando ocorrem os efeitos do tratamento. No caso de pacientes ambulatoriais esta etapa ocorre fora do ambiente hospitalar, e é realizada na maioria das vezes pelo próprio paciente.

\section{Ciclo de Assistência Farmacêutica}

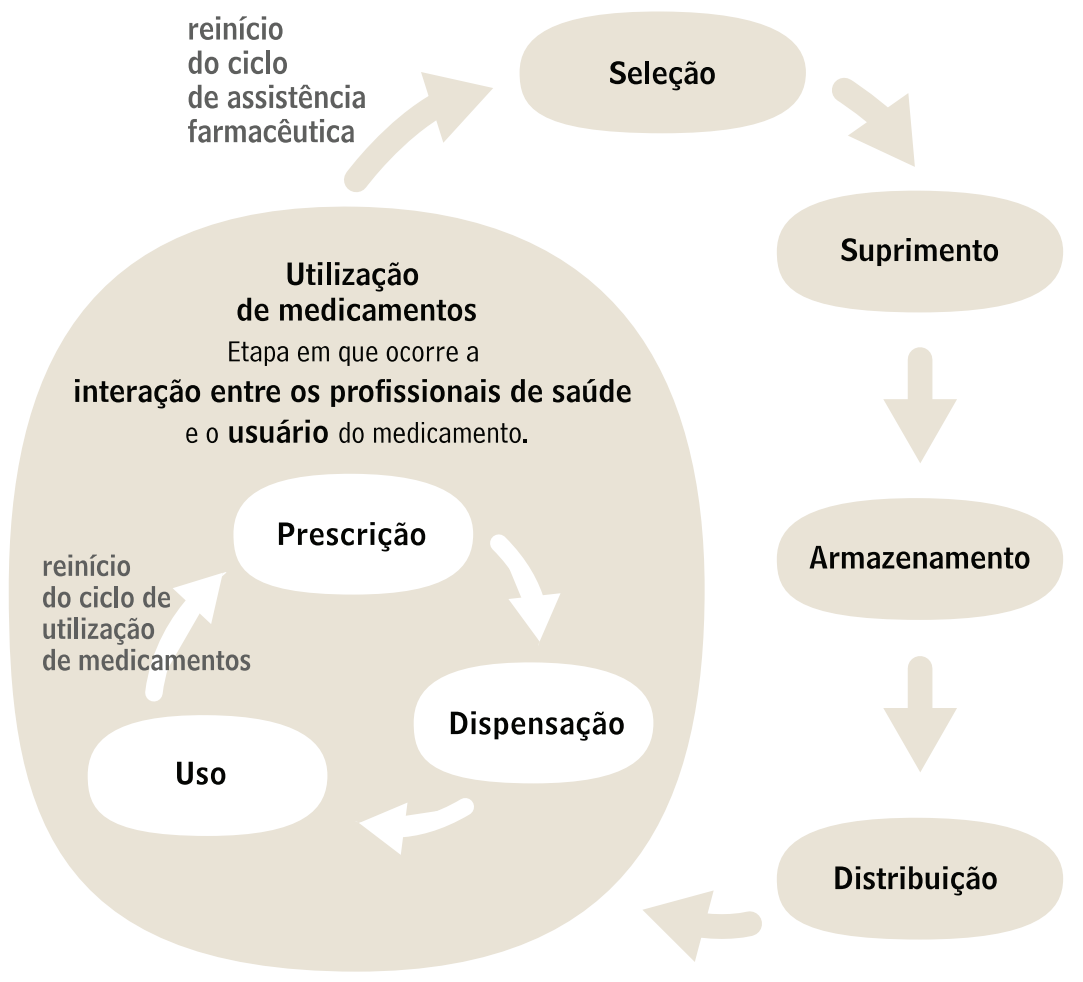

Figura 1: Ciclo de Utilização de Medicamentos no contexto da Assistência Farmacêutica. Lima (2012), a partir de Oliveira, Bermudez e Osório de Castro, 2007.

\section{Metodologia}

Com o objetivo de investigar a auto-administração de medicamentos por pacientes crônicos, foram inicialmente feitas 20 entrevistas não diretivas. As entrevistas foram realizadas com pacientes cardiopatas atendidos em hospital público do munícipio do Rio de Janeiro.

Em seguida foi efetuada a transcrição e análise de conteúdo das entrevistas. Utilizou-se como base teórica para esta etapa Bardin (1979) e Mucchielli (1982).

Após transcrição das entrevistas os trechos relativos ao fenômeno estudado foram selecionados, codificados e organizados conforme as categorias geradas ao longo da análise de conteúdo. (Lima, 2012) 
Com base nos trechos codificados relativos à utilização de medicamentos, foi construído e aplicado questionário piloto. Foram feitas então alterações para melhor adequar o questionário a fatores como: tempo de realização do questionário, vocabulário dos entrevistados e facilidade na anotação da resposta pela pesquisadora.

O questionário final apresentou 5 partes:

- $1^{\mathrm{a}}$ parte: dados gerais e de saúde dos entrevistados

- $2^{\text {a }}$ parte: perguntas relativas à adesão ao tratamento

- $3^{\text {a }}$ parte: perguntas relativas à auto-administração dos medicamentos

- $4^{a}$ parte: perguntas relativas à comunicação na utilização de medicamentos e intervalos entre dosagens

No presente artigo serão apresentadas a $1^{\mathrm{a}}$ e $3^{\mathrm{a}}$ partes: dados dos entrevistados e perguntas relativas à auto-administração dos medicamentos.

\subsection{Aplicação do questionário}

Optou-se pela aplicação do questionário em ambiente não-hospitalar, por ter-se observado, na realização da etapa anterior, que aspectos relativos diretamente à instituição de saúde onde as entrevistas haviam sido realizadas - que não faziam parte do estudo - tendiam a ser o foco das respostas ou influenciá-las.

\subsubsection{Participantes da pesquisa.}

Responderam ao questionário 35 pacientes cardiopatas. O critério para seleção dos entrevistados foi a auto-referencia da morbidade, no qual o paciente informa ao pesquisador ter sido diagnosticado portador do tipo de doença investigada. ${ }^{1}$

A amostragem utilizada foi do tipo não-probabilística acidental (Rudio, 1980, p. 63). Foram, portanto, entrevistadas as 35 primeiras pessoas que declararam ter sido diagnosticadas com cardiopatias e que se disponibilizaram a participar da pesquisa.

1. O critério de morbidade auto-referida para dados relativos a prevalência de doenças crônicas é utilizado na Pesquisa Nacional de Amostra Domiciliar -- PNAD. (Almeida et al, 2002) 


\section{Resultados}

\subsection{Perfil dos respondentes}

Dos 35 participantes, a maioria foi de mulheres. A faixa etária com maior número de entrevistados foi a de pacientes entre 65 e 79 anos.

respondentes por gênero

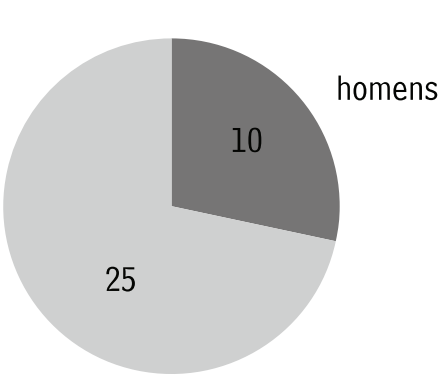

Gráfico 1. respondentes por gênero respondentes por faixa etária

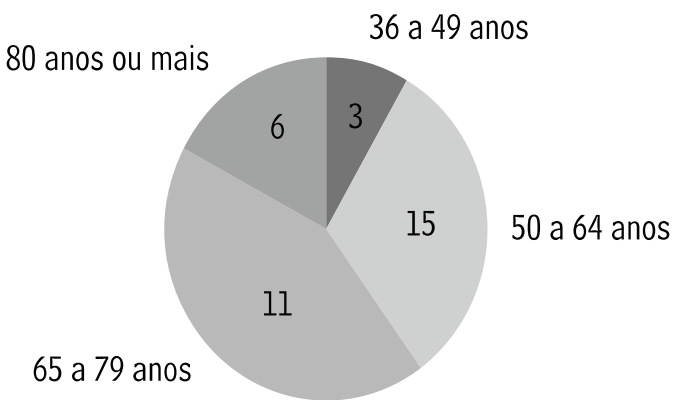

Gráfico 2. respondentes por faixa etária

Quanto à renda mensal, a maioria dos entrevistados declarou receber entre 1 e 3 salários mínimos.

A respeito da escolaridade, a maioria dos entrevistados havia cursado o ensino fundamental e quatro não frequentaram a escola.

respondentes por renda

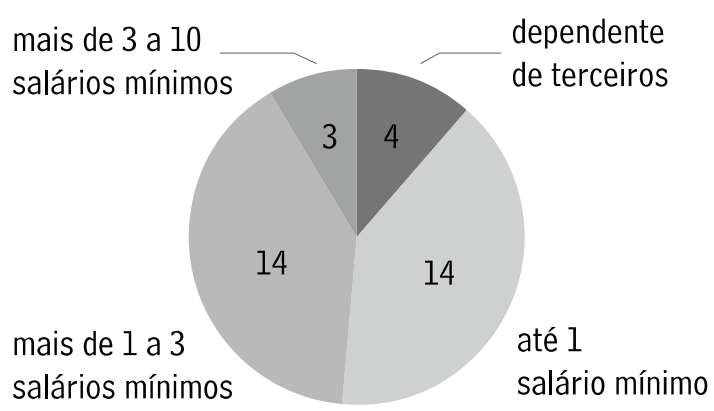

Gráfico 3. respondentes por renda

\section{respondentes por escolaridade}

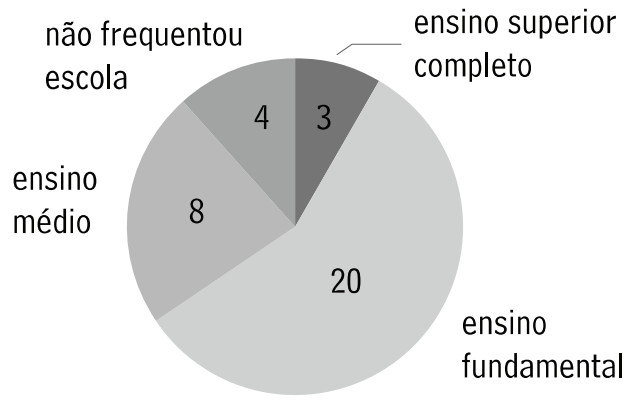

Gráfico 4. respondentes por escolaridade

A maioria dos respondentes utiliza medicamentos para enfermidades crônicas há pelo menos 5 anos. 
Dos 35 entrevistados, 25 declararam ser atendidos em unidades públicas de saúde.

\section{respondentes pelo tempo que toma medicamentos para enfermidades crônicas}

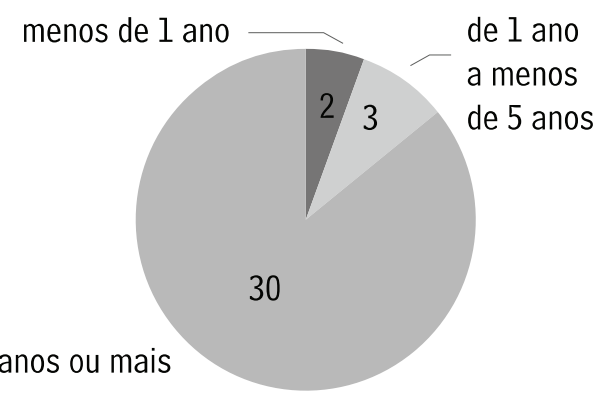

Gráfico 5. respondentes pelo tempo que toma medicamentos para enfermidades crônicas

\section{respondentes pelo tipo de serviço de saúde}

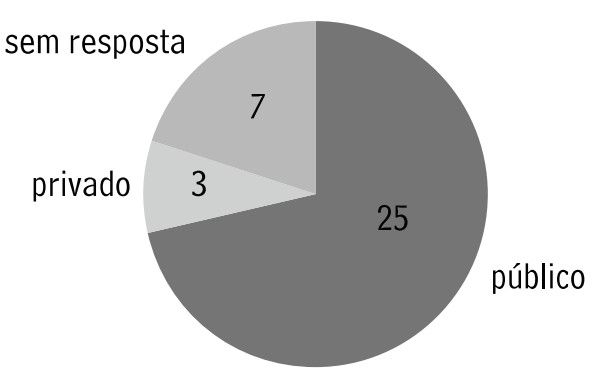

Gráficos 1 a 6. respondentes pelo tipo de serviço de saúde que utiliza

A maior parte dos entrevistados afirmou ser atendida por um profissional de saúde prescritor.

Quanto ao número de medicamentos auto-administrados, 10 respondentes declararam utilizar mais de 6 medicamentos, e somente três respondentes administravam apenas um medicamento.

\section{respondentes pelo número de médicos prescritores}

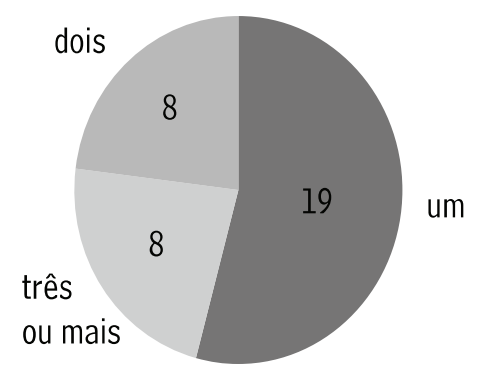

Gráfico 6. respondentes pelo número de médicos prescritores respondentes pelo número de medicamentos diferentes que faz uso

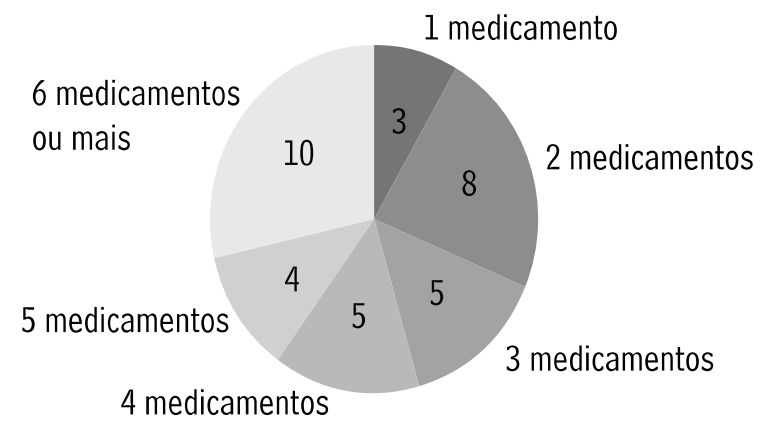

Gráfico 7. respondentes pelo número de medicamentos que faz uso

\subsection{Auto-administração dos medicamentos}

As perguntas apresentadas a seguir correspondem à parte 3 do questionário, relativa à auto-administração de medicamentos. 
Todos os entrevistados tinham um lugar específico para a armazenagem dos medicamentos. Três deles ainda guardam os medicamentos na caixa onde recebiam os remédios da prefeitura, quando esses eram entregues diretamente na casa dos pacientes. A manutenção da caixa para esta finalidade apresenta uma interação do sistema de saúde com o cotidiano do paciente.

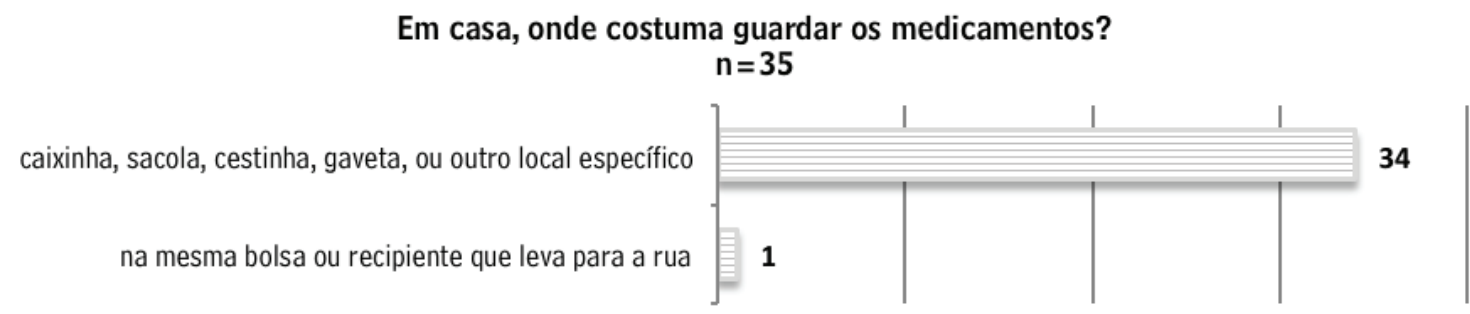

Gráfico 7. Questionários - Respostas à pergunta 7

A pergunta a seguir, ainda relativa à armazenagem dos medicamentos, diz respeito à guarda dos comprimidos que vão ser utilizados. A maioria mantém os comprimidos nas embalagens onde eles são recebidos - na caixa, quando a dispensação ocorre nas farmácias, e na cartela, quando a dispensação se dá nas unidades públicas de saúde. O número de respostas maior do que o de respondentes se dá pelo fato que um mesmo paciente recebe alguns dos medicamentos em caixas e outros diretamente na cartela.

\section{Onde costuma deixar os comprimidos? $\mathrm{n}=35$}

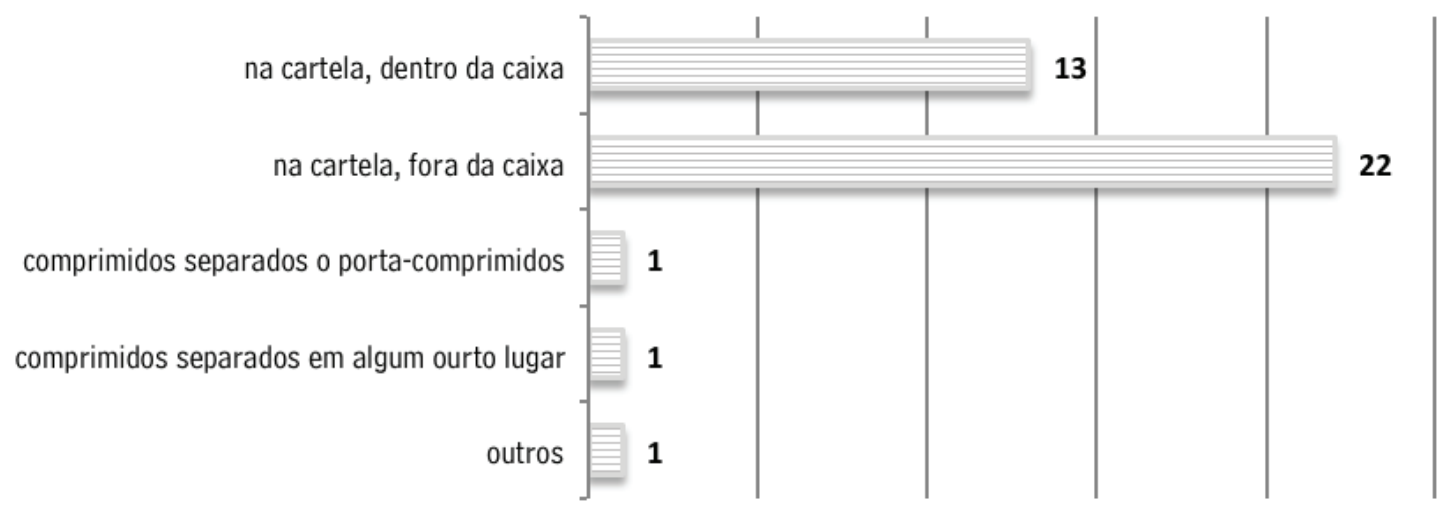

Gráfico 8. Questionários - Respostas à pergunta 8

As duas perguntas seguintes investigam a presença do medicamento no cotidiano do paciente. Observa-se, a partir das repostas obtidas, que o 
medicamento acompanha na rotina da maioria dos pacientes entrevistados, tanto em sua residência como fora dela.

Nota-se ainda que a embalagem do medicamento - cartela/caixa - continua sendo o principal meio de armazenagem dos comprimidos também quando esses são utilizados fora da residência dos entrevistados.

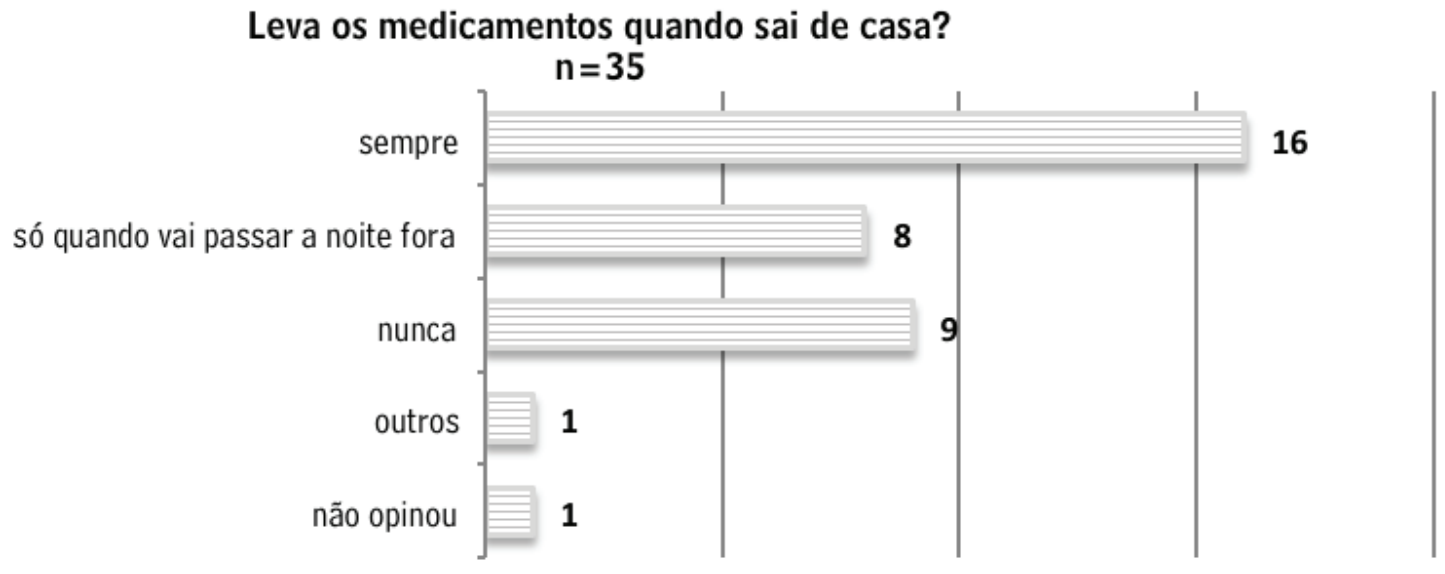

Gráfico 9. Respostas à pergunta 9

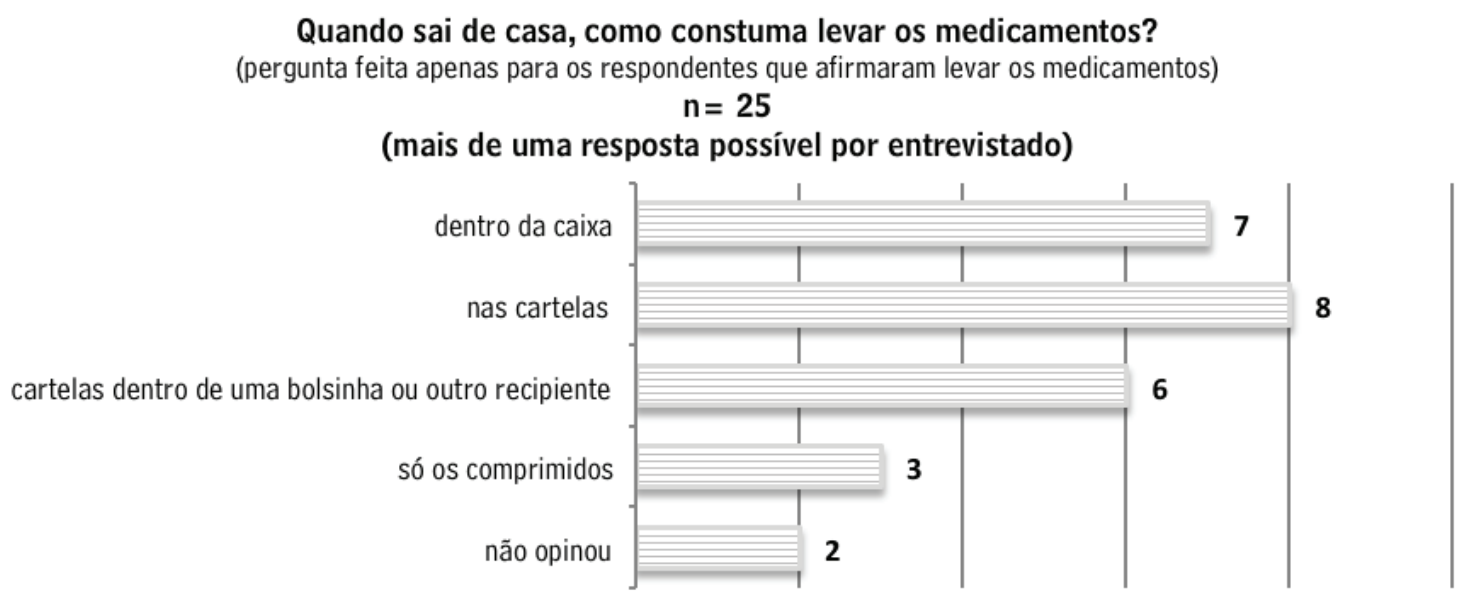

Gráfico 10. Questionários - Respostas à pergunta 9.1

Analisando-se as respostas às próximas duas perguntas, observa-se que a cartela representa um elemento importante na identificação do medicamento. O nome do medicamento é um fator de informação decisivo, mas outros 
elementos gráficos presentes na cartela, somados, representam 27 respostas. As respostas no item "outros" foram: forma do nome e quantidade de comprimidos na cartela. É importante ressaltar que a resposta "forma do nome" é diferente da resposta "nome na cartela". A primeira, dada por uma entrevistada sem escolaridade, significa que é a forma da palavra, e não sua leitura, que é o fator de identificação.

\section{Reconhece as cartelas dos diferentes medicamentos? $\mathrm{n}=35$}

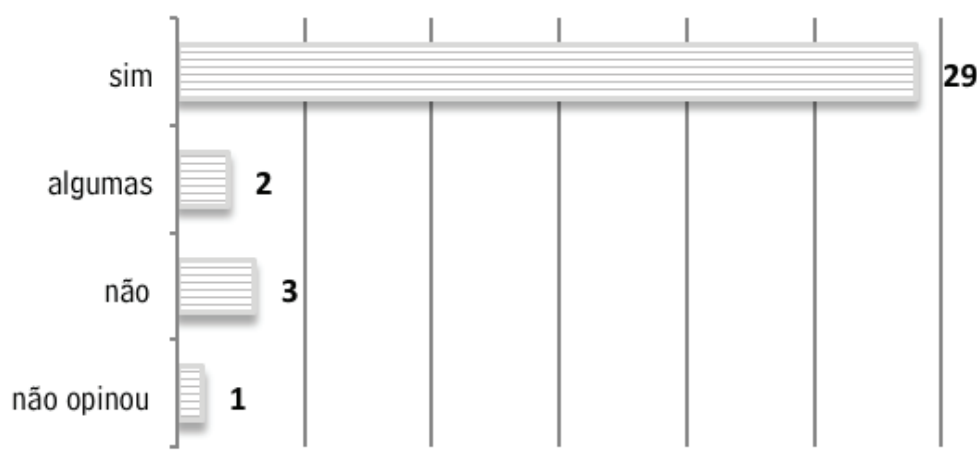

Gráfico 11. Questionário -- respostas à pergunta 10

\section{Como identifica a cartela? \\ (pegunta feta apenas aos respondentes que afirmaram identificar as diferentes cartelas) $\mathrm{n}=31$}

(mais de uma resposta possível por entrevistado)

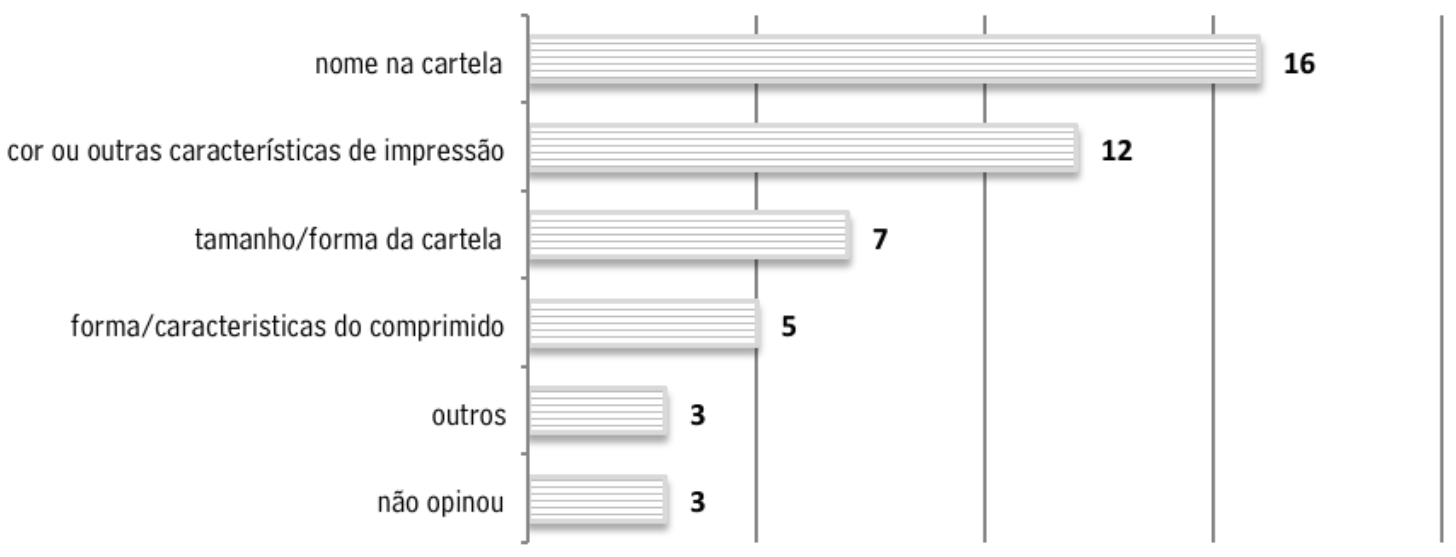

Gráfico 12. Respostas à pergunta 10.1 
Nas respostas a seguir observa-se que o comprimido, mesmo dentro da cartela, também representa um forte elemento de identificação do medicamento. Dos 35 entrevistados, 25 relatam identificar os comprimidos dos diferentes medicamentos administrados e apresentam o tamanho e a cor como os principais fatores de identificação dos medicamentos. Chama a atenção que a ranhura presente no medicamento para facilitar a sua divisão tenha sido apresentada como fator de identificação do medicamento por 5 respondentes. Na categoria "outros" foram apresentados como elementos de identificação: o gosto e um número (possivelmente identificando a dose) impresso no comprimido.

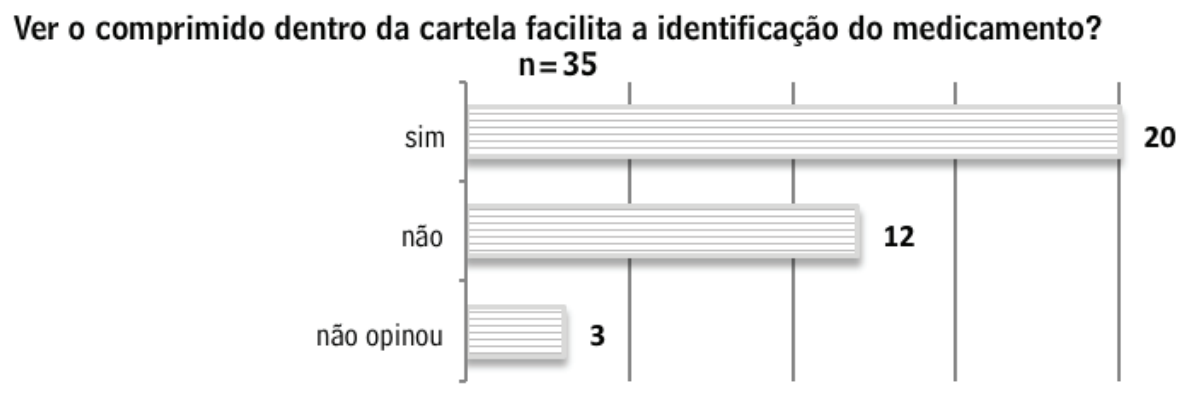

Gráfico 13. Respostas à pergunta 11

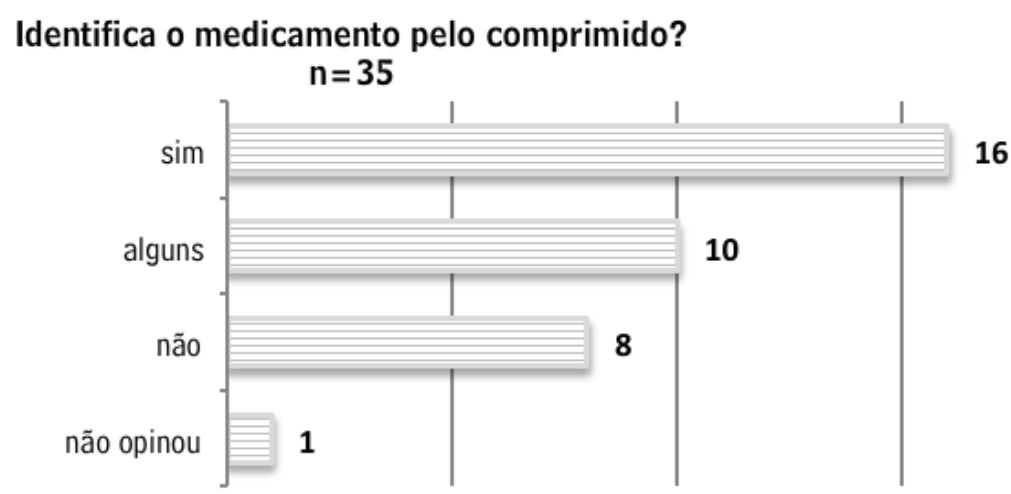

Gráfico 14. Respostas à pergunta 12 


\section{Como identifica o comprimido?}

(pergunta feita apedas aos respondentes que afirmaram

identificar pelo menos um medicameto por características do comprimido)

$\mathrm{n}=\mathbf{2 7}$

(mais de uma resposta possível por entrevistado)

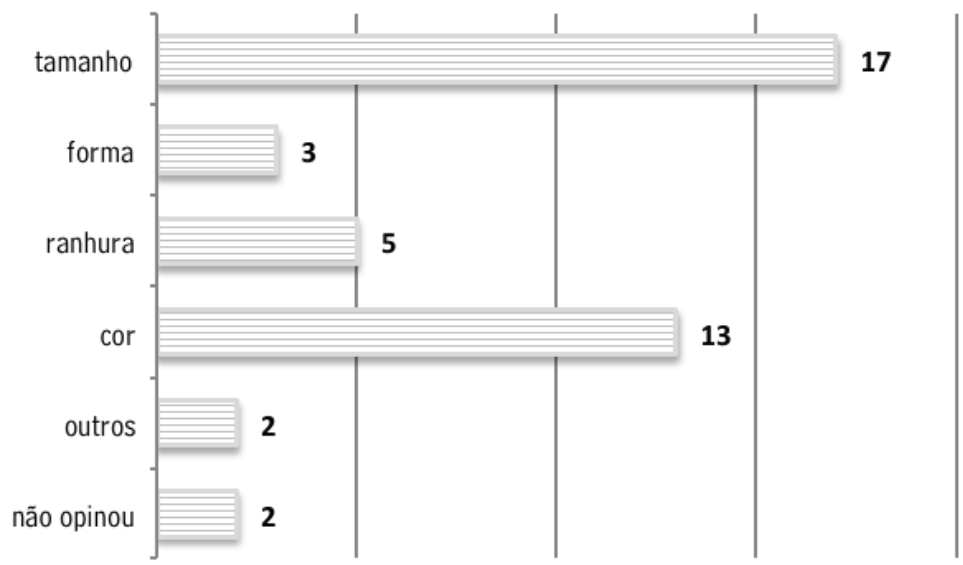

Gráfico 15. Questionários — Respostas à pergunta 12.1

A pergunta a seguir baseou-se na observação, durante a etapa piloto dos questionários, que os pacientes costumavam deixar a receita médica em local visível para consultá-la. O grande número de respostas na opção "outros" indica que houve falha na escolha das opções de respostas apresentadas no questionário. Dentre os locais descritos pelos respondentes estão: junto com os medicamentos (por 9 respondentes) e na bolsa ou junto com documentos (por 5 respondentes). Essas respostas indicam a relevância da receita médica na rotina dos pacientes. Observa-se que o número de respostas à pergunta 13 excede $o$ número de respondentes, pois um dos entrevistados relatou guardar a receita médica em uma pasta, mas também ter uma cópia fixada na porta da geladeira.

Onde guarda a receita médica?

$\mathrm{n}=35$

(mais de uma resposta possível por entrevistado)

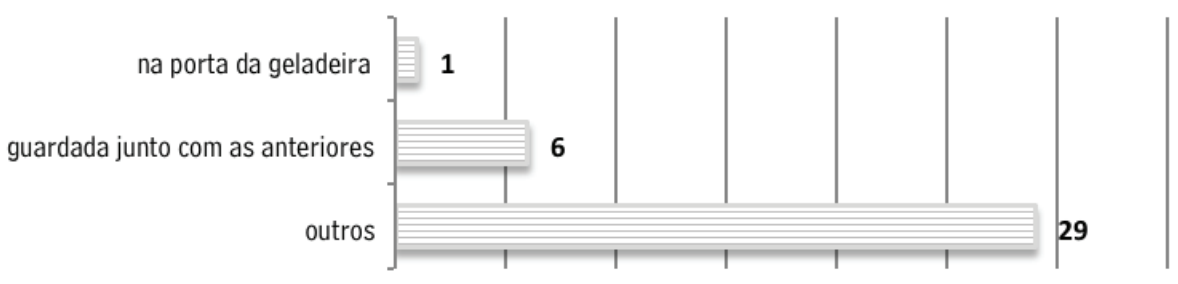

Gráfico 16. Questionários - Respostas à pergunta 13. 
Com relação ao uso da caixinha de porta-comprimidos, parte dos respondentes não a conhece, a maioria conhece e não usa e apenas um respondente afirmou que gostaria de usar. Os motivos apresentados para a não utilização de porta-comprimidos são: o medicamento estar mais seguro na embalagem original, o uso da caixa ser confuso e a tarefa de realocar os comprimidos representar mais trabalho do que a utilização da embalagem original. Um dos respondentes afirmou que a organização dos porta-comprimidos - por dias da semana - gera confusão e sugeriu que a organização fosse feita segundo o nome dos medicamentos.

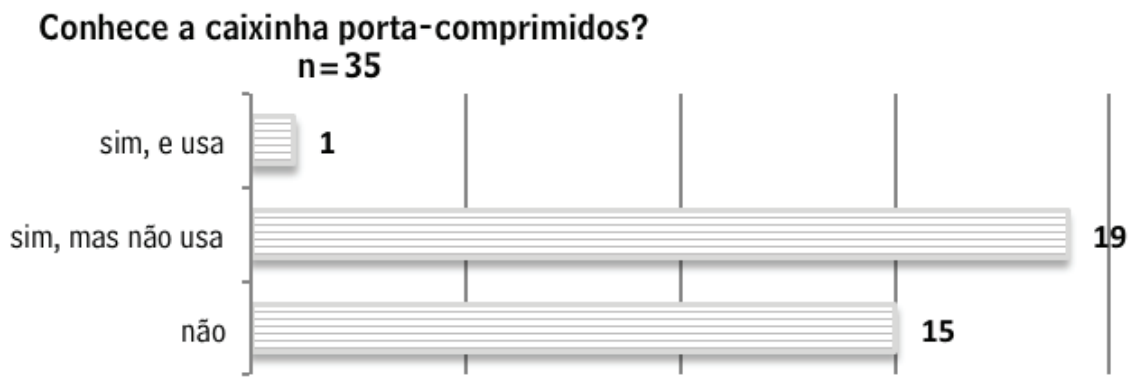

Gráfico 17. Questionários - Resposta à pergunta 14

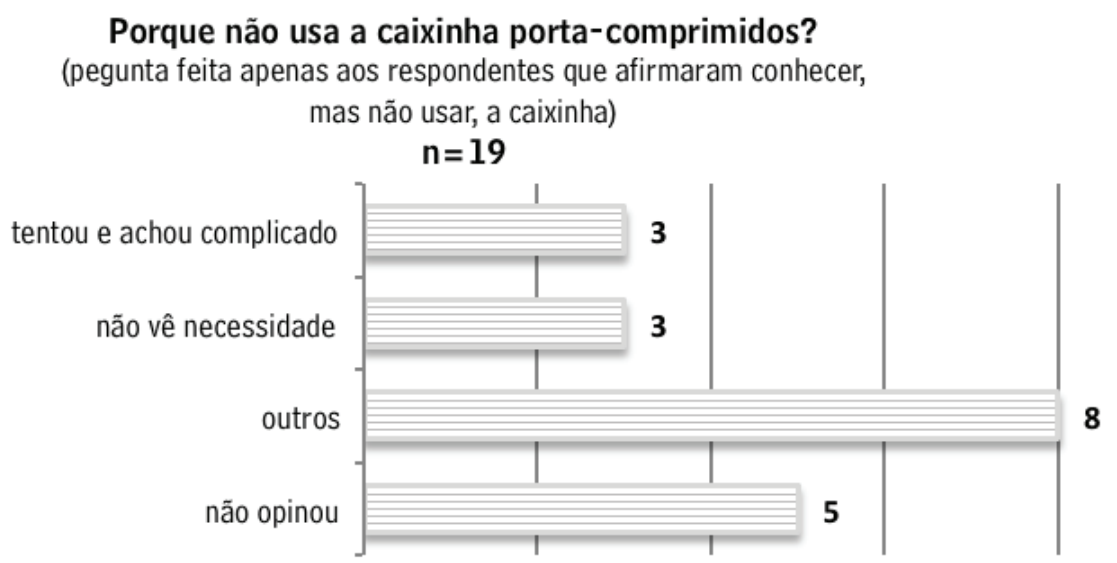

Gráfico 18. Questionários — Respostas à pergunta 14.1 


\section{Conclusões e desdobramentos}

A auto-administração de medicamentos por pacientes crônicos é uma tarefa complexa devido à incidência de comorbidades e, portanto, à diversidade de medicamentos prescritos.

Os pacientes criam estratégias para administrar seus tratamentos com base no seu conhecimento e capacidades individuais.

A partir das respostas obtidas no questionário apresentado, observa-se a relevância de cada elemento presente na utilização de medicamentos - caixas, cartelas, comprimidos e receita médica - na tomada de informação a respeito dos medicamentos auto-administrados.

Destaca-se que o conteúdo escrito (nome do medicamento), ainda que importante, tem menos relevância na identificação dos medicamentos do que a comunicação visual ${ }^{2}$ das embalagens e do próprio comprimido.

A relevância do comprimido como elemento de comunicação visual chama a atenção. Suas características visuais (tamanho, forma, cor, e até mesmo a ranhura para facilitar sua divisão, ou um pequeno número gravado) são informações relevantes para sua identificação. Destaca-se que 20 dos 35 pacientes, relataram que quando é possível ver o comprimido na cartela - ou seja, quando um dos lados da cartela é feito de material transparente - a identificação do medicamento é mais fácil.

A utilização de medicamentos, em especial por pacientes crônicos, representa assunto complexo e vital tanto para a saúde de cada paciente como para a viabilidade do sistema de saúde. Portanto, é relevante que se aprofunde o conhecimento a este respeito, tendo como foco aspectos ergonômicos, ou seja, de interação do paciente / usuário com o sistema de saúde e medicamentos.

Possíveis desdobramentos da presente pesquisa incluem:

- pesquisa qualitativa a respeito dos aspectos da comunicação visual presentes na auto-administração de medicamentos.

- investigação das demais etapas do uso de medicamentos, a fim de contextualizar sua utilização

- análise, no projeto e fabricação de medicamentos, da intencionalidade dos aspectos visuais das embalagens e medicamentos

- investigação da possibilidade de maior colaboração do sistema de saúde na auto-administração consciente dos medicamentos por pacientes crônicos, a partir de objetos que auxiliem os pacientes / usuários

2. Utiliza-se aqui a definição de comunicação visual conforme Bruno Munari, excluindo o sentido do conteúdo escrito na definição do conceito. Segundo o autor "Praticamente tudo o que os nossos olhos vêm é comunicação visual; uma nuvem, uma flor, um desenho técnico, um sapato, um panfleto, uma libélula, um telegrama (excluindo seu conteúdo), uma bandeira" (Munari, 2000, p. 87). 


\section{Bibliografia}

ALMEIDA, Márcia F. et al. Prevalência de doenças crônicas autorreferidas e utilização de serviços de saúde. Ciência \& Saúde Coletiva. v.7, n.4, p.743-756. 2002 .

BARDIN, Laurence. Análise de conteúdo. Lisboa: Edições 70, 1979.

BRASIL, Ministério da Saúde. Portaria $\mathbf{n}^{0}$ 3.916/MS/GM, de 30 de outubro de 1998. Dispõe sobre a aprovação da Política Nacional de Medicamentos. Brasília: Ministério da Saúde, 1998

BRASIL. Ministério da Saúde. A vigilância, o controle e a prevenção das doenças crônicas não transmissíveis: DCNT no contexto do Sistema Único de Saúde brasileiro. Ministério da Saúde, Secretaria de Vigilância em Saúde, Departamento de Análise de Situação de Saúde. Brasília: Ministério da Saudi, 2005.

INSTITUTO BRASILEIRO DE GEOGRAFIA E ESTATÍSTICA. Análise dos resultados da projeção da população do Brasil. In: Estudos e pesquisas, n. 24, Projeção da população do Brasil por sexo e idade 1980-2050. Rio de Janeiro: IBGE, 2009. Disponível em: < http://www.ibge.gov.br/home/estatistica/populacao/indic_sociosaude/2009/indicsaude.pdf >. Acesso em: 16 dez. 2013. LORIG, K. Self-management of chronic illness: a model for the future. Generations. V.17, n.3, p.11-14, outono, 1993

MALTA, Deborah C.; CEZÁRIO, Antônio C.; MOURA, Lenildo de; NETO, Otaliba L de M.; JUNIOR. A construção da vigilância e prevenção das doenças crônicas não transmissíveis no contexto do sistema único de saúde. Epidemiologia e serviços de saúde. Vol. 15, n. 3 jul/set 2006.

MALTA, Deborah C.; MERHY, Emerson E. O percurso da linha do cuidado sob a perspectiva das doenças crônicas não transmissíveis. Interface (Botucatu), v.14, n.34 p.593-605, Epub Set 17, 2010. Disponível em: <http://www.scielo. br/pdf/icse/2010nahead/aop0510.pdf>. Acesso em 6 dez. 2013.

MUCCHIELLI, Roger. A entrevista não-diretiva. Rio de Janeiro: Martins Fontes, 1978a.

MUCCHIELLI, Roger. L'analyse de contenu des documents et des communications. Paris: Co-ed. ESF E.M.E, Lib. Tech., 1982. 
MUCCHIELLI, Roger. O questionário na pesquisa psicossocial. Rio de Janeiro: Martins Fontes, 1979.

MUNARI, Bruno. Design e comunicação visual. Lisboa: Edições 70, 2000.

OLIVEIRA, Maria A.; BERMUDEZ, Jorge A.Z.; OSÓRIO DE CASTRO, Claudia G.S. Assistência farmacêutica e acesso a medicamentos. Rio de Janeiro: Fiocruz, 2007.

ORGANIZAÇÃO MUNDIAL DE SAÚDE. Adherence to long-term therapies: Policy for action, Meeting Report 4-5 June 2001. Genebra: World Health Organization, 2001.

PEPE, Vera L. E.; \& OSÓRIO DE CASTRO, Claudia G. S. A interação entre prescritores, dispensadores e pacientes: informação compartilhada como possível benefício terapêutico. Cadernos de Saúde Púbica. n.16, v.3, p.815-822, jul-set, 2000. Disponível em: <http://www.scielosp.org/pdf/csp/v16n3/2966. pdf.> Acesso em: 10 fev. 2014.

RUDIO, Frans V. Introdução ao projeto de pesquisa. Perópolis: Vozes, 1986.

Rio de Janeiro, Secretaria de Controle Externo: $4^{\mathrm{a}}$ Inspetoria Geral de Controle Externo. Avaliação do Programa Remédio em Casa. Disponível em: <http:// www.tcm.rj.gov.br/Noticias/2119/Cartilha_Remedios_full.pdf> Acesso em 10 fev. 2014. Tribunal de Contas do Município do Rio de Janeiro: Rio de Janeiro, 2005. 
Recebido em: 10/06/2013

Aceito em: 01/10/2013

\section{Como citar}

LIMA, Vera Lopes de Abreu; MORAES, Anamaria de. Relevância da comunicação visual na auto-administração de medicamentos por pacientes crônicos: uma abordagem ergonômica. Arcos Design. Rio de Janeiro: PPD ESDI - UERJ. Volume 7 Número 2 Dezembro 2013. pp. 172-189. Disponível em: [http://www.epublicacoes.uerj.br/index.php/arcosdesign]

DOI

10.12957/arcosdesign.2013.12189

\section{(c) (1) (3)}

A Revista Arcos Design está licenciada sob uma licença Creative Commons Atribuição - Não Comercial - Compartilha Igual 3.0 Não Adaptada. 